\title{
Association between the IL10 rs1800896 Polymorphism and Tardive Dyskinesia in Schizophrenia
}

\author{
Kwang-Yeon Choi ${ }^{1 *}$, Jeong Min Choo ${ }^{2 \star}$, Youn-Jung Lee ${ }^{3}$, Yujin Lee ${ }^{4}$, \\ Chul-Hyun Cho ${ }^{1,5}$, Seung-Hyun $\mathrm{Kim}^{6}$, and Heon-Jeong Lee ${ }^{5,6} \bowtie$ \\ 1Department of Psychiatry, Chungnam National University College of Medicine, Daejeon, Republic of Korea \\ ${ }^{2}$ Department of General Surgery, Korea University College of Medicine, Seoul, Republic of Korea \\ ${ }^{3}$ Department of Psychiatry, Gyeongsang National University School of Medicine, Jinju, Republic of Korea \\ ${ }^{4}$ Department of Psychiatry, Seoul Metropolitan Eunpyeong Hospital, Seoul, Republic of Korea \\ ${ }^{5}$ Chronobiology Institute, Korea University, Seoul, Republic of Korea \\ ${ }^{6}$ Department of Psychiatry, Korea University College of Medicine, Seoul, Republic of Korea
}

Objective Interleukin-10 (IL-10) is a major immunoregulatory cytokine and its gene plays a fundamental role in anti-inflammatory and immunosuppressive activity. This study aimed to examine the association between the IL10 gene promoter -1082G/A polymorphism (rs1800896) and tardive dyskinesia (TD) in schizophrenia.

Methods Two hundred and eighty unrelated Korean schizophrenic patients participated in this study (105 TD and 175 non-TD patients). TD was diagnosed using the Research Diagnostic Criteria for TD and Abnormal Involuntary Movement Scale (AIMS). Genotyping was performed by RT-PCR and high-resolution melting curve analysis.

Results The distributions of genotypic frequencies did not differ between patients with and without TD $\left(\chi^{2}=4.33, \mathrm{p}=0.115\right)$. However, allelic frequencies of the two groups were different $\left(\chi^{2}=4.45, \mathrm{p}=0.035\right)$; the A allele frequency was higher in TD. The total AIMS scores of the three genotypes were not different $(\mathrm{F}=1.33, \mathrm{p}=0.266)$. However, the total AIMS scores of the A allele carrier and the A allele non-carrier were significantly different $(t=5.79, p<0.001)$. Logistic regression analaysis showed that $I L 10-1082 \mathrm{G} / \mathrm{A}$ genotype significantly predicts presence of TD $(\mathrm{p}=0.045)$ after adjusting for covariates such as age and treatment duration.

Conclusion This finding suggests that the A allele of rs1800896 may be associated with TD development following a low IL-10 function.

Psychiatry Investig 2020;17(10):1031-1036

Key Words Tardive dyskinesia, Oxidative stress, Interleukin-10.

\section{INTRODUCTION}

Tardive dyskinesia (TD) is an involuntary, purposeless, and stereotypic movement disorder that develops in some parts of patients who have undergone long-term antipsychotic treatment. For typical antipsychotics, the accumulated prevalence of TD after 1-year treatment is $5 \%$, and the prevalence increases by up to $20 \%$ after long-term treatment. The typical mani-

Received: May 21, 2020 Revised: August 19, 2020

Accepted: September 2, 2020

$\triangle$ Correspondence: Heon-Jeong Lee, MD, PhD

Department of Psychiatry, Korea University College of Medicine, 73 Goryeodae-ro, Seongbuk-gu, Seoul 02841, Republic of Korea

Tel: +82-2-920-6721, Fax: +82-2-929-7679, E-mail: leehjeong@korea.ac.kr

*These authors contributed equally to this work.

(a) This is an Open Access article distributed under the terms of the Creative Commons Attribution Non-Commercial License (https://creativecommons.org/licenses/by$\mathrm{nc} / 4.0$ ) which permits unrestricted non-commercial use, distribution, and reproduction in any medium, provided the original work is properly cited. festations of this neuroleptic-induced disorder are as follows: 1) orofacial movements such as chewing, smacking of lips, protrusion of tongue, ${ }^{1}$ spasmodic blinking of eyes, and grimacing of the face and 2) choreic movements such as sudden and involuntary movements of the limbs. TD patients usually do not recognize these manifestations as serious adverse effects of antipsychotics; they may consider them as their odd habits and ignore them until they become severe. Therefore, psychiatrists need to investigate these symptoms thoroughly.

The etiopathogenic mechanism of TD is not well understood. The conventional hypothesis is that long-term administration of antipsychotics leads to dopamine receptor supersensitivity. However, the hypothesis does not adequately account for all cases of TD. First, although all rodents universally show dopamine supersensitivity following the administration of a dopamine receptor blocker, only $20-25 \%$ of patients who are treated with the first-generation antipsychotics 
have TD. Second, although older patients have decreased capacity for dopamine supersensitivity in their brains, the prevalence of TD increases with age. Third, TD symptoms are irreversible even after withdrawal from the antipsychotics. ${ }^{2}$ Last, while it takes only several days for the number of dopamine binding sites to increase, it takes several months or years for TD to occur in patients who take antipsychotics. ${ }^{1}$

Multiple line of evidence supposed that oxidative stress, imbalance between free radical and antioxidant, and resulting neurodegeneration is etiopathogenetic mechanism of TD. ${ }^{2}$ Long-term exposure to antipsychotics increases dopamine turnover and leads to excessive production of oxidative metabolites. ${ }^{2,3}$ Some studies have postulated that increased lipid peroxidation in the cerebrospinal fluid (CSF) due to imbalances in oxidation mechanisms can lead to cellular dysfunction and apoptosis of neuronal cells in vulnerable brain area such as dopamine-rich basal ganglia.

Old age, female gender, history of brain damage, high-dose antipsychotics, and long-term use of antipsychotics are known risk factors of TD. Excessive production of neurotoxic free radicals in patients treated with chronic antipsychotics exposure related, make vulnerable patient with low capacity eliminating excess free radical ${ }^{1,4}$ have a degenerative change in vulnerable brain area (dopamine-rich basal ganglia) to develop tardive dyskinetia in the vulnerable period (old age). The effort finding genes carrying individual genetic susceptibility was focused in cytochrome P450 (CYP), neurotransmitters such as dopamine and serotonin, and oxidative stress. ${ }^{3,5}$

IL-10 is an important immunoregulatory cytokine that acts on most hematopoietic cell types and enables various functions. IL-10 is also known to have anti-inflammatory and anti-oxidant effects. One of the most important effects is the decrease in TNF- $\alpha$ production to limit oxidative burst and alleviate oxidative stress. ${ }^{6}$ IL-10 suppresses the inflammatory response via cytokines such as TNF- $\alpha$ and oxidative stress. Thus, it decreases the production of reactive oxygen species (ROS). ${ }^{7}$ Previous studies have shown that CSF IL-10 levels increase in schizophrenia patients and that IL10 gene polymorphism is associated with the susceptibility to, and the occurrence of, schizophrenia. ${ }^{8}$

IL10 rs1800896, a single nucleotide polymorphism (SNP) located upstream of the IL10 gene, is the target of this study. According to a study in 2009, A allele of rs1800896 restricts the production of pro-inflammatory cytokines such as TNF- $\alpha$, IL-6, and IL-8 and increases the risk of prostate cancer. ${ }^{9}$ Another study has shown that AA homozygotes of the promotor region in the IL10 gene reduces the production of IL-10. Therefore, reduced production of IL-10 due to AA homozygotes may prevent removal of increased oxidative stress, which makes patients treated with antipsychotics more vulnerable to TD.
Though the prevalence of TD may have decreased after the introduction of the second-generation antipsychotics, ${ }^{2} \mathrm{TD}$ is still one of the most serious delayed, irreversible side effects of chronic antipsychotic use. ${ }^{10}$ Thus primary prevention and early diagnosis of TD is crucial. To predict the occurrence of TD, this study investigated whether A allele of rs 1800896 SNP prevents the production of IL-10, anti-oxidative function, and removal of oxidative stress, which in turn increases the occurrence of TD. This study also investigated whether the existence of the A allele in rs1800896 influences the AIMS scores.

\section{METHODS}

\section{Subjects and assessments}

A total of 280 unrelated Korean schizophrenia patients participated in this study. All the subjects were inpatients enrolled from the three collaborating hospitals of Korea University Hospital. They met the diagnostic criteria of schizophrenia, determined by board-certified psychiatrists using the Korean version of the Structured Clinical Interview for the fourth edition of the Diagnostic and Statistical Manual of Mental Disorders. Subjects with significant neurological comorbidities, mental retardation, substance-related disorders, and other major psychiatric disorders were excluded. This study was approved by the Ethics Committee of the Korea University Hospital (06-2594), and all the participants provided written informed consent. Other characteristics of these subjects have been previously reported. ${ }^{11-15}$

The participants comprised 105 and 175 schizophrenics with and without TD (non-TD), respectively, who were matched for exposure to antipsychotics and other relevant variables. All the non-TD patients had been treated with typical antipsychotics for at least 10 years. The TD sample included 25 patients who had taken atypical antipsychotic drugs and 18 who had been treated for $<10$ years. These differences between the inclusion criteria for the groups were acceptable as the occurrence of TD in patients exposed to antipsychotics for $<10$ years is highly indicative of genetic susceptibility.

All the subjects had taken a stable dosage of antipsychotics for $>3$ months before TD was assessed. TD was diagnosed based on scores on the Abnormal Involuntary Movement Scale (AIMS), which measures the severity of involuntary movements in seven domains on a scale from 0 to 4 . Subjects were diagnosed with TD when they had two or more 2-point ratings or one or more 3-point ratings for the first seven items of AIMS. Psychopathology was assessed using the Positive and Negative Syndrome Scale (PANSS). Other findings related to these subjects have been reported previously. 


\section{Genotyping}

Blood samples (5-10 mL) were collected in EDTA tubes, and genomic DNAs were isolated using a NucleoSpin Blood DNA Extraction Kit (Macherey-Nagel, Germany). The procedure followed the manufacturer's instructions. Genotyping was performed using high-resolution melting curve analysis. ${ }^{16}$ The polymerase chain reaction (PCR) was performed with a $20-\mu \mathrm{L}$ reaction mixture in a 96-well CFX96 real-time PCR system (Bio-Rad Laboratories, Inc., Hercules, CA, USA). The reaction mixture included the following: 1) $2 \mu \mathrm{L}$ of genomic DNA, 2) $200 \mathrm{mM}$ of primer IL10 rs1800896, forward primer 5'-GAC AAC ACT ACT AAG GCT TCT-3', and reverse primer 5'-ATG GAG GCT GGA TAG GAG-3', 3) SsoFast EvaGreen Supermix ( $1 \times$ final concentration; BioRad Laboratories, Inc.), and 4) sterile $\mathrm{H}_{2} \mathrm{O}$. The amplification protocol was as follows. An initial denaturation at $98^{\circ} \mathrm{C}$ for 3 min was followed by 39 cycles of denaturation at $98^{\circ} \mathrm{C}$ for $10 \mathrm{~s}$ and $58^{\circ} \mathrm{C}$ for $20 \mathrm{~s}$. After the initial denaturation at $95^{\circ} \mathrm{C}$ for $10 \mathrm{~s}$ and $65^{\circ} \mathrm{C}$ for $10 \mathrm{~s}$, melting curves were generated from $65^{\circ} \mathrm{C}$ to $95^{\circ} \mathrm{C}$ in increments of $0.2^{\circ} \mathrm{C}$ at each cycle. Melting profiles were analyzed with the Precision Melt Analysis software (BioRad Laboratories, Inc.).

\section{Statistical analysis}

The Hardy-Weinberg equilibrium was tested using the chisquare $\left(\chi^{2}\right)$ test for goodness of fit. Categorical data were also analyzed using the $\chi^{2}$ test, and the differences between continuous variables were evaluated using the analysis of variance.
Logistic regression analysis was used to determine if independent variable such as genotype of IL10 rs1800896 could account for TD (nominal dependent variable) after adjusting for confounding factors such as age, sex and medication duration. IL10 rs1800896 genotype was coded according to the number of $\mathrm{G}$ allele ( $\mathrm{AA}=0, \mathrm{AG}=1, \mathrm{GG}=2$ ) based on additive effect model. The analyses were performed using SPSS version 18.0 for Windows (SPSS Inc., Chicago, IL, USA). All statistical analyses were two-tailed, and the level of statistical significance was set at $\mathrm{p}<0.05$.

\section{RESULTS}

Of the 280 participants, 105 and 175 were included in the TD and non-TD groups, respectively. Table 1 shows the demographic data of the participants. Table 2 presents the genotypic and allelic frequencies of IL10 gene -1082G/A polymorphism. The distributions of the genotypic frequencies $\left(\chi^{2}=\right.$ $4.328, \mathrm{p}=0.115)$ of TD and non-TD patients were similar. However, the allelic frequencies $\left(\chi^{2}=4.45, \mathrm{p}=0.035\right)$ of TD and nonTD patients demonstrated a statistically significant difference.

Table 3 shows the differences between the AIMS scores stratified by genotypes. There were no significant differences between the AIMS scores of the three genotype groups. Of the 280 subjects, 272 were A allele carriers (AA+AG) and 5 were non-carriers (GG). Table 4 shows the differences between the AIMS scores of the A allele carriers and non-carriers. The A allele carriers generally had higher AIMS scores than non-

Table 1. Descriptive statistics for demographic data of schizophrenic patients with and without TD

\begin{tabular}{|c|c|c|c|c|}
\hline & Schizophrenia without TD $(\mathrm{N}=175)$ & Schizophrenia with TD $(\mathrm{N}=105)$ & T or $\left(\chi^{2}\right)$ & $\mathrm{p}$ \\
\hline $\operatorname{Sex}(M / F)$ & $93 / 82$ & $55 / 50$ & $(0.0153)$ & 0.901 \\
\hline Age (years) & $43.47 \pm 9.17$ & $46.29 \pm 9.72$ & -2.43 & 0.016 \\
\hline Duration of illness (years) & $18.81 \pm 6.57$ & $19.95 \pm 8.56$ & -1.12 & 0.264 \\
\hline Treatment duration (years) & $17.48 \pm 6.12$ & $15.74 \pm 7.74$ & 1.892 & 0.060 \\
\hline AIMS orofacial (1-4) & $0.41 \pm 0.84$ & $5.36 \pm 2.96$ & -16.57 & $<0.001$ \\
\hline AIMS extremities (5-6) & $0.26 \pm 0.57$ & $1.64 \pm 1.75$ & -7.73 & $<0.001$ \\
\hline AIMS trunk (7) & $0.01 \pm 0.08$ & $0.54 \pm 0.96$ & -5.69 & $<0.001$ \\
\hline AIMS total $(1-7)$ & $0.75 \pm 1.06$ & $7.67 \pm 4.22$ & -16.36 & $<0.001$ \\
\hline
\end{tabular}

TD: tardive dyskinesia, AIMS: Abnormal Involuntary Movement Scale

Table 2. Comparison between the IL10 -1082G/A (rs1800896) genotype and allele frequencies of schizophrenic patients with and without TD

\begin{tabular}{lcccccccc}
\hline & \multicolumn{4}{c}{ Genotypes } & & \multicolumn{3}{c}{ Allele frequencies } \\
\cline { 2 - 4 } \cline { 6 - 8 } & AA & AG & GG & & A & G \\
\hline Schizophrenia without TD (N=175) & 146 & 24 & 5 & $\chi^{2}=4.328$ & & 0.90 & 0.10 & $\chi^{2}=4.45$ \\
Schizophrenia with TD (N=105) & $83.4 \%$ & $13.7 \%$ & $2.9 \%$ & & & & \\
& 95 & 10 & 0 & $\mathrm{p}=0.115$ & 0.95 & 0.05 & $\mathrm{p}=0.035$ \\
& $90.5 \%$ & $9.5 \%$ & $0 \%$ & & & & \\
\hline
\end{tabular}

TD: tardive dyskinesia 
carriers; they had statistically significant higher orofacial (14 ), trunk (7), and total (1-7) AIMS scores, but not extremities (5-6) AIMS scores.

Although the sample size was small, there were fewer AG heterozygotes than AA homozygotes; the GG homozygotes were even fewer than AG heterozygotes. There was no GG homozygote among the schizophrenic patients with TD (Table 2). Sensitivity analysis performed by grouping AA vs. AG+GG showed no group difference of AIMS total score and subscores between $\mathrm{AA}$ and $\mathrm{AG}+\mathrm{GG}$ groups.

We performed binary logistic regression analysis to elucidate whether IL10 -1082G/A genotype predict presence or absence of TD after adjusting for covariates such as age, sex and treatment duration. The model predicted that as individuals had more $\mathrm{G}$ alleles $(\mathrm{AA}=0, \mathrm{AG}=1, \mathrm{GG}=2)$, the likelihood to having TD significantly decreased $(\mathrm{OR}=0.473, \mathrm{p}=0.045)$ (Table 5).

\section{DISCUSSION}

Several studies have emphasized on the association between genetic polymorphism and TD and found an association between TD and polymorphisms in genes related to 5-HT and dopamine receptors. ${ }^{5}$ IL-10 is well known for its anti-inflammatory and anti-oxidant properties. Previous studies have reported that the SNP in IL10 reduces IL-10 production. There are data supporting the antipsychotic-related increase in oxidative stress in the brain and the relationship between oxidative stress and the pathogenesis of TD. The above-mentioned

Table 3. Comparison between the AIMS scores of the IL10 -1082G/A (rs1800896) genotype groups

\begin{tabular}{lcccc}
\hline & & Genotypes & & \\
\cline { 2 - 4 } & AA (N=239) & AG (N=33) & GG (N=5) & 0.292 \\
AIMS orofacial (1-4) & $2.32 \pm 3.09$ & $2.06 \pm 3.08$ & $0.20 \pm 0.45$ & 0.653 \\
AIMS extremities (5-6) & $0.80 \pm 1.38$ & $0.64 \pm 1.03$ & $0.40 \pm 0.55$ & 0.661 \\
AIMS trunk (7) & $0.22 \pm 0.66$ & $0.15 \pm 0.57$ & $0.00 \pm 0.00$ & 0.266 \\
AIMS total (1-7) & $3.45 \pm 4.39$ & $2.82 \pm 3.86$ & $0.60 \pm 0.89$ & \\
\hline
\end{tabular}

AIMS: Abnormal Involuntary Movement Scale

Table 4. Comparison between the AIMS scores and clinical variables of the carriers of $A$ allele (AA+AG) and the $A$ allele non-carriers (GG) of IL10 -1082G/A (rs1800896)

\begin{tabular}{|c|c|c|c|c|}
\hline & \multicolumn{2}{|c|}{ Carrier } & \multirow[b]{2}{*}{ T or $\left(\chi^{2}\right)$} & \multirow[b]{2}{*}{$\mathrm{p}$} \\
\hline & $\begin{array}{l}\text { A allele carrier }(\mathrm{AA}+\mathrm{AG}) \\
\mathrm{N}=275\end{array}$ & $\begin{array}{l}\text { A allele non-carrier (GG) } \\
\qquad \mathrm{N}=5\end{array}$ & & \\
\hline $\operatorname{Sex}(M / F)$ & $146 / 129$ & $2 / 3$ & $(-0.58)$ & 0.563 \\
\hline Age (years) & $44.54 \pm 9.47$ & $44.00 \pm 10.27$ & 0.117 & 0.913 \\
\hline Duration of illness (years) & $19.15 \pm 7.31$ & $15.80 \pm 9.01$ & 1.189 & 0.297 \\
\hline Treatment duration (years) & $16.90 \pm 6.79$ & $14.20 \pm 6.38$ & 0.883 & 0.378 \\
\hline AIMS orofacial (1-4) & $2.29 \pm 3.09$ & $0.20 \pm 0.45$ & 7.619 & $<0.001$ \\
\hline AIMS extremities (5-6) & $0.78 \pm 1.35$ & $0.40 \pm 0.55$ & 0.635 & 0.526 \\
\hline AIMS trunk (7) & $0.21 \pm 0.65$ & $0.00 \pm 0.00$ & 0.724 & 0.470 \\
\hline AIMS total (1-7) & $3.37 \pm 4.32$ & $0.60 \pm 0.89$ & 5.794 & $<0.001$ \\
\hline
\end{tabular}

AIMS: Abnormal Involuntary Movement Scale

Table 5. Binary logistic regression analysis of $I L 10$ gene polymorphism (-1082G/A; rs1800896) and tardive dyskinesia

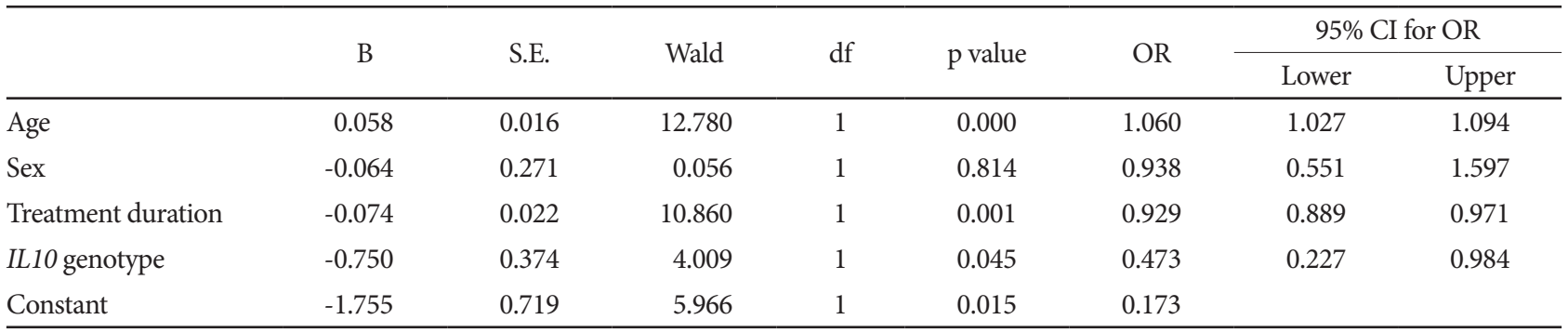

OR: adds ratio, CI: confidence interval 
findings and data facilitated our study on the association between the etiopathogenic mechanisms of TD and rs1800896 of ILI0.

IL-10 prevents TNF- $\alpha$ production and oxidative stress. The findings from our study support those of previous studies that the A allele of rs 1800896 reduces IL-10 production and antiinflammatory and anti-oxidant reactions and inhibits alleviation of oxidative stress and neurotoxicity due to long-term administration of antipsychotics. This suggests that decreased IL-10 production causes critical damage to tissues, such as cardiac muscle or myelin sheath. Hence, the SNP of rs1800896 may reduce IL-10 production and increase oxidative stress and vulnerability to TD.

According to Capsoni et al., ${ }^{17} \mathrm{IL}-10$ influences the inflammatory response of neutrophils. IL-10 inhibits oxidative burst by reducing lipopolysaccharide (LPS)-induced cytokine production of neutrophils, which suggests that A allele reduces IL-10 production, increases LPS-induced cytokine production from neutrophils, prevents removal of oxidative burst, and increases vulnerability to TD. As Table 2 demonstrates, the G allele reduces the susceptibility to schizophrenia, given that AG heterozygotes and GG homozygotes are markedly fewer than AA homozygotes. The absence of GG homozygotes in TD patients further underscores this postulation. However, this study is limited by the small sample size. If the sample was larger, statistically significant data may have been obtained.

Although long-term administration of atypical (second-generation) antipsychotics can also cause TD, typical antipsychotics are known to cause TD much more frequently. Therefore, atypical antipsychotics have become the primary drug of choice for schizophrenia. However, the efficacies of the two classes of antipsychotics are similar, and metabolic syndrome is a critical side effect of atypical antipsychotics. Furthermore, since typical antipsychotics are cheaper than their atypical counterparts, they are more widely used in clinical settings. ${ }^{5}$ Therefore, it is important to predict high-risk groups who have genetically susceptible factors such as SNP; then, drug substitutions can be done before administration; otherwise, early diagnostic tools for TD are required urgently.

Small sample size and vicinity of GG genotype is limitation of our study. Extremely low frequency of G allele of IL-10 Sparsity of GG allele of IL10 rs1800896 in the east asian (GG genotype $<1 \%$ of population) has limited the collection of sample sufficient to draw more conclusive conclusions. Further research is needed in other ethnics with a high frequency of GG alleles.

A previous study on the association between oxidative stress and TD highlighted that the antioxidant vitamin E ( $\alpha$-tocopherol) initially seemed to prevent and treat early phases of TD, but it showed low or no efficacy in longstanding TD. However, the accumulation of study data that support the association between oxidative stress caused by antipsychotics and TD provides the foundation for exploring the clinical application of antioxidants for treatment and prevention of $\mathrm{TD}^{2} . \mathrm{In}$ addition to the prevention of $\mathrm{TD}$, modalities for preventing progression of TD and facilitating the recovery of patients are crucial.

\section{Acknowledgments}

This study was supported by the Korea Health 21 R\&D Project funded by the National Research Foundation of Korea (2019R1A2C2084158 and 2017M3A9F1031220)

\section{Conflicts of Interest}

The authors have no potential conflicts of interest to disclose.

\section{Author Contributions}

Conceptualization: Heon-Jeong Lee, Seung-Hyun Kim. Data curation: Jeong Min Choo, Youn-Jung Lee. Formal analysis: Kwang-Yeon Choi, Jeong Min Choo, Yujin Lee. Funding acquisition: Heon-Jeong Lee. Investigation: Kwang-Yeon Choi, Jeong Min Choo, Yujin Lee. Methodology: Kwang-Yeon Choi, Jeong Min Choo, Yujin Lee, Youn-Jung Lee. Project administration: Heon-Jeong Lee, Chul-Hyun Cho. Resources: Heon-Jeong Lee, Chul-Hyun Cho. Software: Kwang-Yeon Choi, Jeong Min Choo. Supervision: Heon-Jeong Lee, Seung-Hyun Kim. Validation: Heon-Jeong Lee, Seung-Hyun Kim. Visualization: Youn-Jung Lee. Writing_original draft: Kwang-Yeon Choi, Jeong Min Choo. Writing_-review \& editing: Heon-Jeong Lee.

\section{ORCID iDs}

Kwang-Yeon Choi https://orcid.org/0000-0001-7134-8723

Jeong Min Choo https://orcid.org/0000-0002-6059-5064

Youn-Jung Lee https://orcid.org/0000-0003-4208-7039

Yujin Lee https://orcid.org/0000-0002-7459-4935

Chul-Hyun Cho https://orcid.org/0000-0002-1663-9680

Seung-Hyun Kim https://orcid.org/0000-0002-2189-4600

Heon-Jeong Lee https://orcid.org/0000-0002-9560-2383

\section{REFERENCES}

1. Cadet JL. Tardive Dyskinesia and Oxidative Stress. In: Connor JR, Editor. Metals and Oxidative Damage in Neurological Disorders. New York (NY): Springer, 1997, p.315-324.

2. Lohr JB, Kuczenski R, Niculescu AB. Oxidative mechanisms and tardive dyskinesia. CNS Drugs 2003;17:47-62.

3. Lee HJ, Kang SG. Genetics of tardive dyskinesia. Int Rev Neurobiol 2011; 98:231-264.

4. Korean Neuropsychiatric Association (Korea). Textbook of Neuropsychiatry 2nd Ed. Seoul; Jungang Moonhwa \& Co.; 2005.

5. Lee $\mathrm{HJ}$, Cho $\mathrm{CH}$. Oxidative stress and tardive dyskinesia: pharmacogenetic evidence. Prog Neuropsychopharmacol Biol Psychiatry 2013;46: 207-213.

6. Moore KW, Malefyt RDW, Coffman RL, O'Garra A. Interleukin-10 and the interleukin-10 receptor. Annu Rev Immunol 2001;19:683-765.

7. Huet O, Laemmel E, Fu Y, Dupic L, Aprico A, Andrews KL, et al. Interleukin 10 antioxidant effect decreases leukocytes/endothelial interaction induced by tumor necrosis factor $\alpha$. Shock 2013;39:83-88.

8. Sun H, Wang F, Fan H, Yan Q, Cui K, Yuan W, et al. The interaction of polymorphisms of IL10 and DBH was associated with general symptoms of PANSS with TD in Chinese Han schizophrenic patients. PLoS ONE 2013:8:e70963.

9. Wang M, Helzlsouer KJ, Smith MW, Hoffman-Bolton JA, Clipp SL, Grinberg V, et al. Association of IL10 and other immune response- and obesity-related genes with prostate cancer in CLUE II. Prostate 2009;69:874- 
885.

10. So SJ, Kang SG, Lee HJ, Kim L. Association between tardive dyskinesia and polymorphisms of TNF- $\alpha$ gene in Korean schizophrenia patients. Korean J Schizophr Res 2013;16:38-42.

11. Son WY, Lee HJ, Yoon HK, Kang SG, Park YM, Yang HJ, et al. GABA transporter SLC6A11 gene polymorphism associated with tardive dyskinesia. Nord J Psychiatry 2014;68:123-128.

12. Park YM, Kang SG, Choi JE, Kim YK, Kim SH, Park JY, et al. No evidence for an association between dopamine D2 receptor polymorphisms and tardive dyskinesia in Korean schizophrenia patients. Psychiatry Investig 2011;8:49-54.

13. Lee HJ, Kang SG, Choi JE, Park YM, Lim SW, Rhee MK, et al. No evidence for association between tyrosine hydroxylase gene Val81Met polymorphism and susceptibility to tardive dyskinesia in schizophrenia. Psychiatry Investig 2009;6:108-111.
14. Lee HJ, Kang SG, Choi JE, Paik JW, Kim YK, Kim SH, et al. No association between dopamine $\mathrm{D} 4$ receptor gene-521 C/T polymorphism and tardive dyskinesia in schizophrenia. Neuropsychobiology 2007;55:47-51.

15. Lee HJ, Kang SG, Paik JW, Lee MS, Cho BH, Park YM, et al. No evidence for an association between $\mathrm{G}$ protein $\beta 3$ subunit gene C825T polymorphism and tardive dyskinesia in schizophrenia. Hum Psychopharmacol 2007;22:501-504.

16. Vossen RH, Aten E, Roos A, den Dunnen JT. High-resolution melting analysis (HRMA): more than just sequence variant screening. Hum $\mathrm{Mu}$ tat 2009;30:860-866

17. Capsoni F, Minonzio F, Ongari AM, Carbonellil V, Galli A, Zanussi C. Interleukin-10 down-regulates oxidative metabolism and antibody-dependent cellular cytotoxicity of human neutrophils. Scand J Immunol 1997;45:269-275. 